\title{
El tema del conocimiento como construcción social: una mirada contemporánea*
}

\author{
José Arlés Gómez**
}

Recibido: 29 de octubre de 2010 Revisado: 3 de diciembre de 2010 Aprobado: 10 de febrero de 2011

\section{Resumen}

En la actualidad las redes de conocimiento constituyen las máximas expresiones del ser humano como productor y reproductor de saberes y conocimientos, además de su necesidad de intercambiar y transferir lo que se aprende y se crea a partir de la interacción social en el contexto de plataformas tecnológicas específicas. Por otro lado, en la época contemporánea, el apoyo a las redes investigativas, así como el soporte a la investigación en las áreas temáticas involucradas, buscan una mejor utilización de los recursos humanos y materiales disponibles en las instituciones, procurando generar masas críticas que puedan permitir nuevas oportunidades de mejora y avance en los espacios alternativos que ofrecen las mismas redes de conocimiento como construcciones sociales.

\section{Palabras clave}

Red social, conocimiento, sociedad, ciencia, tecnología, ser humano. 


\title{
The Theme of Knowledge as Social Construction: a Contemporary Vision
}

\author{
José Arlés Gómez
}

\begin{abstract}
In the twenty-first century global world, knowledge networks are the best expressions of the human being as producer and player of knowledge and expertise, in addition to its need to exchange and transfer what is learned and what is created from the social interaction in the context of specific technology platforms. On the other hand, in contemporary times, support for research networks, as well as support for research in the subject areas involved, seek a better use of available human and material resources in the institutions that could generate critical masses of enabling new opportunities for improvement and progress in the alternative spaces that offer the same knowledge networks as social constructions.
\end{abstract}

\section{KEYWORDS}

Social network, knowledge, society, science, technology, human being. 


\section{INTRODUCCIÓN}

El programador y fundador del concepto Software Libre y de la Free Software Fundation, Richard Stallman ${ }^{1}$ formula la siguiente pregunta: “¿qué necesita la sociedad actual respecto al tema del conocimiento?", a este cuestionamiento responde el mismo Stallman: "necesita información que esté realmente disponible para todos los ciudadanos". En efecto, existe una enorme preocupación por inventar programas que las personas puedan examinar, corregir, acomodar y optimizar para una mejor utilización. Este ejemplo del sofware libre emerge como una pequeña puerta de desarrollo que adquiere fuerza y logra constituirse como una línea potencial de creación de conocimiento y desarrollo tecnológico en la época contemporánea. Ya el paradigma de la era industrial es reemplazado por el de la revolución tecnológica. Del cultivo de la memoria humana, se pasa al cultivo de la I.A².

Con respecto a la I.A. es bueno recordar que hasta los mismos sistemas inteligentes deben ser dotados con mecanismos de

1 Richard Matthew Stallman (Nueva York, 1953) es principalmente conocido por el establecimiento de un marco de referencia política, ética y legal para el movimiento del software libre, como una alternativa al desarrollo y distribución del software no libre o privativo. Es también inventor del concepto de copyleft (aunque no del término), un método para licenciar software de tal forma que su uso y modificación permanezcan siempre libres y queden en la comunidad. Entre sus logros notables como programador incluyen la participación en el editor de texto GNU Emacs, el compilador GCC, y el depurador $\mathrm{GDB}$, bajo la rúbrica del Proyecto GNU.

2 Se denomina inteligencia artificial (I.A.) a la parte de las ciencias de la computación dedicada al desarrollo de agentes racionales no vivos. El concepto "agente", se entiende como cualquier elemento capaz de percibir su entorno (recibir entradas), procesar tales percepciones y actuar en su entorno (proporcionar salidas), y entiéndase a la racionalidad como la característica que posee una elección de ser correcta, más específicamente, de tender a maximizar un resultado esperado (este concepto de racionalidad es más general y por ello más adecuado que inteligencia para definir la naturaleza del objetivo de esta disciplina). retroalimentación que les permitan tener conocimiento de estados internos, igual que sucede con los humanos que disponen de propiocepción ${ }^{3}$, interocepción ${ }^{4}$ y nocicepción ${ }^{5}$. Esto es fundamental tanto para tomar decisiones como para conservar su propia integridad y seguridad. La retroalimentación en sistemas está particularmente desarrollada en cibernética, por ejemplo en el cambio de dirección y velocidad autónomo o la cantidad de memoria disponible para una operación determinada.

Por otro lado, Fernando Robles, sociólogo chileno, asevera que los procesos de "descomposición y recomposición de las identidades de los actores sociales se desenvuelven y son observables en el terreno de las interacciones cotidianas que le otorgan significación a las relaciones entre los actores sociales" (Robles, 1999, p. 314). Los nuevos códigos, las nuevas formas de interpretar el conocimiento y las nuevas semánticas crean amplias posibilidades de construcción social de la misma realidad, a la vez que se van buscando los mejores mecanismos para legitimar y objetivar dichas prácticas. Un ejemplo claro de estas afirmaciones es la proliferación de espacios

3 La propiocepción es el sentido que informa al organismo de la posición de los músculos. La información es transmitida al cerebro a través de los husos musculares, localizados en el interior de los músculos. Estos husos están compuestos de pequeñas fibras musculares (fibras intrafusales) inervadas por nervios que informan de la longitud del músculo. Sin la propiocepción seríamos incapaces de movernos en la oscuridad o de percibir la posición de nuestras extremidades (Wikipedia).

4 La interocepción es el sentido que nos informa de los estados de nuestras vísceras (sensación de hambre, sensación de sed); sus receptores sensoriales se localizan dentro del propio cuerpo (Javier Echegoyen)

5 La nocicepción (también conocida como nociocepción o nocipercepción) es el proceso neuronal mediante el que se codifican y procesan los estímulos nocivos." 1 . Se trata de la actividad aferente producida en el sistema nervioso periférico y central por los estímulos que tienen potencial para dañar los tejidos (Wikipedia). 
virtuales con foros de opinión, espacios públicos de acceso a internet, ferias de instalación de programas nuevos y espacios en donde se hace sentir la misma opinión y hasta las protestas de la sociedad civil contemporánea, que comprenden desde las huelgas generales hasta los movimientos cívicos como Hazteoír o Voota, que parecen enfocarse hacia reivindicaciones bastante particulares en temas políticos y de cultura ciudadana.

\section{Antecedentes de los ESTUDIOS SOBRE REDES SOCIALES DEL CONOCIMIENTO}

Con el advenimiento de la cultura mediática y de la información periódica en las sociedades contemporáneas, el mito de la organización social, evoluciona a un nuevo paradigma, es la primera vez que una institución social se da a la tarea de autodescribirse de manera sistemática y actualizada. De esta forma, los medios de comunicación se convierten entre los siglos XIX y XX en el mecanismo de autorrelato social más profundo jamás visto en época alguna de la historia. Efectivamente, nunca antes el hombre había dispuesto de autoimágenes del mundo sociocultural con tanta regularidad y velocidad, con un ahorro de tiempo y espacio tan enormes y en donde las mismas miradas y prácticas humanas se ven forzosamente convocadas a un replanteamiento profundo de sus identidades, redes de comunicación y vivencias colectivas. Desde esta perspectiva se va generando una gran preocupación por profundizar en el estudio de las llamadas "redes sociales de conocimiento".

Se consideran como pioneros del estudio de las redes sociales, en el siglo XVIII, a
Durkheim y a Tönnies. Para el primero, los hechos y fenómenos sociales, nacen cuando las personas que interactúan componen una realidad que ya no se explica en términos de cualidades o propiedades individuales, sino colectivas. Para Durkheim se debe dar paso a una sociedad moderna desde el concepto de "solidaridad orgánica", la cual despliega reciprocidad y participación entre los diversos actores que componen la misma sociedad. Según Tönnies, los grupos sociales se pueden establecer o bien como vínculos formales e instrumentales o como vínculos compartidos por medio de creencias y valores.

Hacia 1954, J.A. Barnes, de la Universidad de Manchester, comenzó a utilizar sistemáticamente el término "redes sociales", para mostrar modelos de parentesco y de lazos socioafectivos, incluyendo los conceptos tradicionalmente utilizados por los científicos sociales: grupos familiares, etnias, subgrupos culturales, etc. Por esta misma época, emergen como pioneros de la construcción social del conocimiento, los denominados "constructivistas", especialmente desde el campo de la psicología y la pedagogía, entre los que se destacan los plateamientos de Piaget (1952), Vygotsky (1978), Ausubel (1963), y Bruner (1960); es importante aclarar que aunque ellos mismos no se denominaron constructivistas, sus teorías y planteamientos claramente aportan las bases para hablar de una construcción social del conocimiento. Entre los planteamientos de dichos pensadores, se cree que los acontecimientos y fenómenos sociales, se desenvuelven concretamente en contextos socioculturales. Desde el mismo constructivismo las construcciones sociales se conciben habitualmente como subproductos de iniciativas humanas al tratar de comprender $y$ 
percibir las propias dinámicas sociales, lo que se denomina como "realidad social", es una construcción siempre en evolución dinámica, pues es reproducida por las personas que interactúan desde sus interpretaciones y conocimientos.

En 1966, Berger y Luckmann escriben una obra denominada La construcción social de la realidad, en ella afirman que el mismo conocimiento se produce merced a las interacciones sociales, al incluir el más básico, que es el "sentido común". Las mismas instituciones sociales se construyen dentro de lo que las sociedades llaman "realidad objetiva" y desde ellas, el mismo conocimiento es negociado y es esencialmente activo y dinámico.

Posteriormente, algunos investigadores contemporáneos, entre los que se encuentra Linton Freeman, quien en su obra The Development of Social Network Analysis (2006) describe la historia de cómo ha sido el progreso de las redes sociales a la vez que hace un análisis de las mismas. Por otra parte, son de destacar los estudios hechos por la Universidad de Chicago, desde los análisis cuantitativos, entre los que se destacan Galaskiewicz, Griswold y Laumann y el grupo de la Universidad de Michigan a la cabeza de Nanlin y Rogers. En el área de habla española se creó en 1998 la revista y la web denominada Redes a partir de la Conferencia Internacional de Redes, que se llevó a Cabo en Barcelona.

Por otro lado, Pier Paolo Donati, sociólogo de la Universidad de Bolonia, en Repensar la Sociedad (2006) propone un nuevo modo de examinar los fenómenos sociales. Se trata de observar la sociedad como "sociedad relacional". Esto implica repensar la sociedad para resolver los problemas culturales, polí- ticos y económicos, haciendo más humanas las formas de organización social propias del siglo XXI. Donati analiza, especialmente, las nuevas formas de configuración de la sociedad y sobre todo políticas sociales en materia de comunicación y relación de las nuevas intersubjetividades humanas.

\section{Definición, CARACTERísticas E IMPLICACIONES SOCIALES DE LAS REDES SOCIALES DE CONOCIMIENTO}

Resulta bastante complicado intentar dar una definición unívoca de lo que son las redes sociales de conocimiento, pues se trata relativamente de un concepto novedoso en el contexto del lenguaje tecnológico, el cual se encuentra profundamente emparentado con las concepciones sociológicas del pasado y presente histórico de la ciencia y del hombre. No obstante, se asume que tal concepto está relacionado con lo tecnológico y lo social, como categorías claves dentro de una visión integral de la definición misma de "redes sociales". Esta combinación entre lo tecnológico y lo social remite al tema del enfoque de la tecnología como sistema, y por ende, del concepto de red de conocimiento.

Según Osorio (2002) en el enfoque sistémico se entiende a la tecnología, no dependiente de la ciencia o representada por el conjunto de artefactos, sino como producto de una unidad compleja, de los que forman parte los materiales, los artefactos y la energía, así como los agentes que la transforman (Quintanilla, 2001). Así las cosas, el factor fundamental del desarrollo tecnológico lo constituye la innovación social y cultural, la cual involucra no sólo a las tradicionales referencias al mercado, sino también a los 
aspectos organizativos, y al ámbito de los valores y de la misma cultura ${ }^{6}$.

\section{Afirman Beltrán y Castellanos que:}

...el concepto de "red de conocimiento" se extiende a una comunidad de personas que, de modo formal o informal, ocasionalmente, a tiempo parcial o de forma dedicada, trabajan con un interés común y basan sus acciones en la construcción, el desarrollo y la compartición mutuos de conocimientos (Beltrán, 2004, p. 1).

Según Seufert las redes de conocimiento son las "redes que se establecen entre los individuos, los grupos y las organizaciones donde no simplemente son importantes las relaciones bilaterales, sino la integridad de las actividades desempeñadas por la propia red de conocimientos" (Rodríguez, Araujo \& Yulianow, 2003, p. 4).

Para Casas dichas redes "implican tanto la formación de redes profesionales y de entrenamiento, como de redes de difusión y transmisión de conocimientos o de innovaciones, que estarían dando lugar a la formación de espacios regionales de conocimiento" (Casas, 2001, p. 21).

Como se puede observar en las anteriores enunciaciones existe la posibilidad de agrupar ciertas particularidades en torno a la importancia del concepto, por un lado, las redes sociales de conocimiento plantean

6 Según Quintanilla, definir la palabra "tecnología" se ha vuelto algo complejo y sujeto a un conjunto de relaciones y de puntos de vista diversos. No presenta el mismo significado en el presente que en el pasado, y no son las mismas respuestas que se dan sobre la tecnología en el uso corriente. Un empresario, por ejemplo, invocaría a las máquinas y a las herramientas como los testimonios de lo que es la tecnología, es decir, aparatos, mecanismos, los cuales tienen utilidad, sirven para algo. Pero si la pregunta recae en el director de un laboratorio de investigación y desarrollo, la respuesta podría ser otra y sin embargo estamos, en principio, hablando de lo mismo, en este caso, la tecnología sería ciencia aplicada. un nuevo paradigma en las relaciones humanas en términos de cooperación y unión organizacional y trabajo en equipo, en la indagación de objetivos mediatizados por el compartimiento de información y desarrollo de procesos de conocimiento. Además, tales redes poseen la tendencia a asociarse con otras redes fuera del contexto donde se inician y multiplican a medida que esta relación avanza en el tiempo y se mejora su interconexión tecnológica.

No obstante, en un contexto más social y dinámico, las redes sociales de conocimiento son las interacciones humanas en la producción, almacenamiento, distribución, transferencia, acceso y análisis de los conocimientos producidos por el hombre de manera sistemática, ya sea por la investigación o por el interés personal o grupal por compartir datos de cualquier índole y a través de cualquier medio, habitualmente electrónicos; con el objeto de desarrollar sus capacidades de creación, entendimiento, poder, estudio y transformación de la realidad que lo rodea en un ámbito territorial y en un contexto económico social determinado.

Según las anteriores definiciones, las redes de conocimiento, desde una valoración objetiva desde su complejidad e historicidad operan a partir de los siguientes elementos primordiales:

- Desde las expresiones de la interacción humana en un contexto social propio y profundamente unido al desarrollo de las sociedades actuales.

- Desde una intencionalidad: promover, almacenar y distribuir conocimiento científico por medio de cualquier método de transmisión tecnológica. 
- Desde el objetivo de dicha transmisión, no sólo desde la información y difusión, sino desde la transformación del entorno en la búsqueda constante del enriquecimiento intelectual del ser humano en su quehacer innovativo y creativo a través del estudio sistemático que brinda un tipo de investigación trans y multidisciplinar.

- Desde su localización en ámbitos históricos, espaciales y territoriales determinados, es decir, que las mismas han existido desde la propia creación del hombre y funcionan en contextos locales, regionales, nacionales e internacionales específicos.

- Desde su relación con la ciencia en el contexto económico social del capitalismo como su máxima expresión y vía de expansión más rápida.

- Desde su tendencia a propagarse y a virtualizarse en el contexto de una sociedad dinámica caracterizada por la pretensión de conocimiento y de globalización.

Según estos contextos se puede decir que las redes de conocimiento se han desarrollado en la época actual gracias a las siguientes razones contextuales:

- A la dinámica de la sociedad globalizada en su interés por llevar a cabo procesos de comunicación del conocimiento a nivel global dentro de una visión de libre mercado.

- Por las mismas particularidades de los sistemas de ciencia y tecnología que generan y mercadean información constantemente. Así como por capacidad de expansión vertiginosa de los sistemas de comunicación de datos y transferencia de imágenes en tiempo real.

- Por la celeridad y bajos precios en el registro de los datos en formatos digitales, dándole más fluidez y accesibilidad a dicha información a nivel mundial.

- Por el advenimiento de la llamada "cultura de la información" y la reorientación del campo laboral en la sociedad del conocimiento, así como por el aumento cada vez más gradual de las inversiones de capital en el estudio tecnocientífico de la información como nuevo medio de expansión del sistema económico imperante.

- Por la reestructuración de la ciencia en cuanto a la predisposición de unificarse y ser más productiva en el análisis práctico de las necesidades humanas con fines a la vez sociales y comerciales, así como por la imperante necesidad de renovación de las organizaciones que producen conocimientos científicos convenientes, según nuevos modelos de gestión más efectivos y eficaces.

Por otra parte, el concepto de "redes sociales de conocimiento" lleva implícita una evocación antropológica debido al interés de producir y transferir la información como consecuencia de la actividad humana, de la misma ciencia que produce y de las transformaciones que generan.

De la misma manera, las redes sociales de conocimiento colocan el acento en el papel de la investigación científica como eje básico de la producción de comprensiones y saberes socialmente válidos, concentrados mayormente en los centros investigativos 
e instituciones educativas universitarias o desde los programas de investigación y desarrollo de los Estados, con ayuda de la empresa privada.

Por su parte Manuel Baeza, sociólogo chileno, en Los caminos invisibles de la realidad social. Ensayo de sociología profunda sobre imaginarios sociales (2000), hace un análisis sobre imaginarios sociales en donde las imágenes socialmente compartidas por las colectividades dan sentido al entorno existencial humano, de tal manera que colocan en evidencia lo intangible, además de situar en los ejes espacio-temporales las creaciones de la realidad desde nuevas miradas intersubjetivas.

Por último, según Martínez quien propone el llamado "paradigma emergente de la ciencia", que según él "debe brotar de la dinámica y dialéctica histórica de la vida humana y se impone, cada vez más con fuerza y poder convincente, a nuestra mente inquisitiva" (Martínez, 1997, p. 156). De hecho, la ciencia es consecuencia de la racionalidad e inteligencia humana y es el ser humano el único capaz de cambiar sus propias interpretaciones sobre la realidad a fin de impulsar cambios en la ciencia moderna. El paradigma emergente, según este autor, descansa en los siguientes postulados científicos.

- El conocimiento y las nuevas ciencias deben fundamentarse en una antropología sistémica, donde el hombre sea el centro del saber bajo parámetros de totalidad e interrelación de los fenómenos que explica. El viejo fundamento de la ciencia a partir de bases artesianas debe ser superado por una visión interdisciplinaria integral donde el método hermenéutico sea la guía para la personalidad científica del hombre que investiga.

- El conocimiento y los saberes descansan en el orden de los sistemas abiertos como respuesta a la causalidad y simplicidad de la ciencia tradicional. Esto hace que los conocimientos científicos se entiendan bajo una totalidad integral unida y no disgregada que interactúa constantemente con la realidad que los produce, los establece y los impulsa.

- El paradigma emergente concibe el conocimiento personal como una nueva visión que incita al entendimiento dialéctico entre el objeto y el sujeto y fundamentalmente del contexto sociohistórico que rodea las interpretaciones teóricas subjetivas del sujeto que investiga.

Desde los anteriores principios del paradigma emergente, Martínez afirma que la metacomunicación y la autorreferencia, hacen que el espíritu crítico-reflexivo del hombre sea transmitido a través de procesos de comunicación, sociales e institucionales, capaces de difundir su esfuerzo para el cuestionamiento y el análisis constante de los fenómenos que estudia.

\section{LAS REDES SOCIALES EN INTERNET}

A inicios del 2001 nacen los primeros sitios en internet que promueven redes de amigos y familiares; para el 2003 aparecen sitios virtuales como son Myspace, Tribe y Friendster. De manera vertiginosa, muchas empresas se adhieren a las redes sociales, 
pues ven ellas un potencial económico y cultural bastante grande, como en el caso de Google quien en el 2004 crea la iniciativa de Orkut, red diseñada para permitir relaciones de amistad, contactos comerciales y relaciones amorosas, según centros de interés, preferencias gastronómicas, religiosas y sexuales de las comunidades que se afilian. En estas redes, como en la gran mayoría, el funcionamiento gira alrededor de un soporte técnico que se actualiza constantemente para ofrecer bienes y servicios a partir de la teoría de grafos ${ }^{7}$.

La gran novedad que disparó el tema de las redes en internet fue la propuesta de Facebook, red que nació en el 2004 de la mano de Mark Zuckerberg, originalmente como propuesta enfocada a la comunidad universitaria, y que se calcula para la fecha cuenta con alrededor de 500 millones de miembros. Es importante también mencionar otras redes de amplia difusión como son Twiter ${ }^{8}$, Youtube y Myspace, igualmente con muchos millones de usuarios y miembros activos.

En términos generales, las redes sociales en Internet contribuyen a viabilizar la conformación de lazos comunicativos según los centros de interés de las poblaciones beneficiarias de la misma; a la vez que integra a millones de personas en la época de la glo-

7 También llamada teoría de las gráticas, estudia las propiedades de los grafos o gráficas como conjuntos de objetos llamados vértices 0 nodos y una selección de pares de vértices, llamados aristas. Característicamente, un grafo se representa mediante una serie de puntos (los vértices) conectados por líneas 0 aristas.

8 Nacida en el 2006, Twitter, tiene su sede en California y es una red social y de microblogging de servicios, que permite a sus usuarios enviar y leer mensajes de llamadas tweets. Los tweets se basan en texto de mensaje de 140 caracteres que aparecen en el perfil del usuario correspondiente. Ha ganado gran popularidad en el mundo entero y se calcula que 200 millones de personas son usuarios de la misma, generando 65 millones de tweets por día y con más de 800 mil consultas de búsqueda por día. balización y planetización del conocimiento; asimismo, facilita los vínculos comerciales y afectivos entre personas de diversa procedencia y nacionalidad. Por otra parte, a través de diversas investigaciones transdisciplinarias, se ha evidenciado que las redes sociales en internet, ayudan a estrechar los lazos culturales, económicos, políticos y familiares, además de contribuir con el entretenimiento y manejo tecnológico de la misma información. Igualmente hay estudios que muestran el peligro de la adicción al "ciberespacio", la pérdida de la intimidad y del control sobre ciertos tipos de información que se considera "confidencial" $\mathrm{y}$ reservada al fuero personal ${ }^{10}$.

\section{Conclusiones}

Se puede concluir, en primera instancia, que las sociedades contemporáneas, llamadas "sociedades de las tecnologías de la informática y la comunicación", se han caracterizado por una batalla basada en el intento de supresión del tiempo con respecto al espacio, lo cual implica una nueva reconstrucción de los discursos sobre el espacio-tiempo que en última instancia promueven una desterritorialización de la vivencia de los grupos sociales y sus relaciones comunicativas.

9 Numerosas investigaciones, dan cuenta de que millones de personas son adictas a los grupos o redes sociales en internet. Desde la obsesión por el Facebook o por el Twiter o los juegos online, así como por el chat, varios expertos en las adicciones a la tecnología informática, afirman que muchas algunas patologías asociadas son la soledad, al autismo, a la depresión y a las relaciones afectivas fracturadas o insuficientes, y pueden contribuir a que se acreciente este fenómeno de preocupante propagación alrededor del mundo

10 Hace relativamente poco Facebook se vio forzada a anular la normatividad tendiente al control de la información de los usuarios de esta red social. La nueva postura de Facebook, actualmente es solicitar la colaboración de sus millones de usuarios para redefinir dichas políticas de empresa. Aquí se observa un ejemplo palpable de la importancia de la presión que pueden ejercer las redes sociales. 
Por otro lado, se puede concluir que las redes sociales de conocimiento reposan en las distintas organizaciones humanas creadas para tal propósito; las mismas son capaces de sistematizar coherentemente las ideas, asignar recursos, aplicar métodos sistematizados y alcanzar efectos conducentes a la invención y desarrollo de las distintas áreas del quehacer social, esto responde al carácter institucionalizado y colectivo de la ciencia. No obstante, dichas redes reconocen también el carácter individualista creador del hombre en lo que se conoce como "ciencia popular" o conocimiento no científico, importante en el flujo de información en dicha red. Los mismos saberes científicos, desde nuevas interpretaciones, deben estructurarse bajo el principio de complementariedad del conocimiento, la antigua visión particular debe sustituirse bajo una visión sistémica interdisciplinaria, es decir, del esquema hipotético deductivo a un esquema sistémico integral, pregonado desde varias corrientes de pensamiento, entre ellas, por el pensamiento complejo.

Por otro lado, es importante tener en cuenta la mediación de las tecnologías de la información y la comunicación en la sociedad contemporánea, que influyen en los nuevos movimientos sociales; de la misma manera, dichas tecnologías de la información y la comunicación contribuyen a afirmar y mantener las conexiones y las nuevas redes de conocimiento y participación social emergentes, las cuales se organizan y desarrollan en función de sus estructuras y capacidades para establecer nuevos paradigmas de información que faciliten la acción colectiva mediante una articulación tecnológica que establece un diálogo dinámico entre los nuevos mundos del conocimiento y las nuevas dinámicas sociales. Con rela- ción a las redes sociales del conocimiento que se han generado a través de internet, cada vez más concurridas, se puede decir que permiten actualizar permanentemente la información tanto personal como grupal, ayudan a visibilizar a muchas poblaciones y grupos humanos que previamente se han mantenido en el anonimato, han permitido la creación de nuevos enlaces a través de la prestación de servicios de diversa índole y han contribuido a acentuar el fenómeno de globalización y planetización de la sociedad contemporánea, así como la capacidad de convocatoria colectiva para apoyar causas humanitarias o contribuir con la exigencia de derechos personales y sociales. A su vez, no se puede desconocer las limitaciones y ciertas desventajas que posee su uso inadecuado en términos de adicciones, pérdida de la privacidad y en ocasiones, manipulación de la información, tanto de las personas como de los mismos grupos sociales.

Por último, desde la evolución que han tenido las redes sociales del conocimiento, especialmente en las últimas dos décadas, se ve la imperiosa necesidad de hacer estudios cada vez inter y transdisciplinarios que ayuden a una mejor comprensión de los fenómenos culturales, psicológicos y sociales que subyacen en la raíz misma de dichas redes. El tema de la cooperación y participación activa de millones de personas en la construcción colectiva del conocimiento, genera grandes retos e incógnitas, implicando la reflexión sobre cómo crear políticas justas e inclusivas, frente a la dinámica de innovación, transmisión y difusión del mismo conocimiento, que no debe quedar en manos de unos pocos grupos económicos fuertes sino que debe favorecer la inserción cada vez más grande de personas que tradicionalmente se han sentido excluidas 
socialmente. De igual manera, se deben repensar los nuevos paradigmas que en términos de bienestar, desarrollo sostenible, cooperación y solidaridad, deben generar

\section{REFERENCIAS}

Baeza, M. (2000). Los caminos invisibles de la realidad social. Ensayo de sociología profunda sobre imaginarios sociales. Chile: RIL Editores.

Casas, R. (2001). La formación de redes de conocimientos. Una perspectiva regional desde México. México: Editorial Anthropos - IISUNAN.

Crook, C. (1996). Computers and the Collaborative Experience of Learning. New York: Routledge.

Daniels, H. (2003). Vygotsky y la pedagogía. Barcelona: Ediciones Paidós.

Donati, P. (2006). Repensar la sociedad. Madrid: Ediciones Internacionales Universitarias.

Linton, F. (2006). The Development of Social Network Analysis. Vancouver: Empirical Press. las nuevas redes sociales del conocimiento, especialmente, desde los países más pobres y excluidos de las tecnologías y avances científicos contemporáneos.

Martínez, M. (1997). El paradigma emergente. Hacia una nueva teoría de la racionalidad humana, 2ª ed. México: Editorial Trillas.

Osorio, C. (2002). Enfoques sobre la tecnología. Revista Iberoamericana de Ciencia, Tecnología, Sociedad e Innovación.

Quintanilla, M. (1988). Tecnología: un enfoque filosófico. Madrid: Fundesco.

Stallman, R. (2001). Por qué el Software no debería tener propietarios. Revista Novática Matellán, Vicente \& González, Jesús \& Heras, Pedro \& Robles, Gregorio (Eds.) (2004). Sobre software libre. Compilación de ensayos sobre software libre. Recuperado de [http://gsyc.escet.urjc. es/ grex/sobre-libre/libro-libre.pdf].

Wasserman, S. \& Faust, K. (1994). Social Network Analysis: Methods and Applications. Cambridge: Cambridge University Press. 